\title{
Atteinte rénale du syndrome d'activation macrophagique
}

\author{
Une cause rare de défaillance multiviscérale
}

\section{Renal Involvement in Hemophagocytic Syndrome A rare cause of multi-organ failure}

\author{
A. Karras \\ Reçu le 11 mars 2018; accepté le 17 mai 2018 \\ (c) SRLF et Lavoisier SAS 2018
}

Résumé Le syndrome d'activation macrophagique (SAM) ou syndrome hémophagocytaire est une pathologie compliquant soit certains déficits immunitaires d'origine génétique, soit certaines maladies hématologiques (essentiellement des lymphomes non hodgkiniens), infectieuses (herpèsvirus, bactéries, parasites) ou auto-immunes (lupus, maladie de Still). Il se caractérise par une suractivation aiguë du système immunitaire et plus particulièrement des lymphocytes $T$ cytotoxiques et des cellules histiocytaires/macrophagiques, déclenchant une production massive de cytokines proinflammatoires avec fièvre, pancytopénie, organomégalie, altérations du bilan hépatique et de la coagulation. Une défaillance multiviscérale est fréquente, nécessitant la prise en charge en réanimation et mettant souvent en jeu le pronostic vital, avec une mortalité qui reste dans certains cas à près de $50 \%$. Le SAM implique souvent le rein, par le biais d'une nécrose tubulaire aiguë, d'une néphropathie interstitielle inflammatoire ou d'une glomérulopathie sévère, responsable de syndrome néphrotique. La mise en évidence de ce syndrome n'est pas toujours facile chez un patient avec un tableau de sepsis ou de choc septique, mais elle peut guider la prise en charge thérapeutique, notamment l'initiation d'un traitement par chimiothérapie ou immunosuppresseurs, selon l'étiologie identifiée.

Mots clés Syndrome d'activation macrophagique Hémophagocytose lymphohistiocytaire · Insuffisance rénale aiguë

\footnotetext{
A. Karras $(\bowtie)$

Service de néphrologie, hôpital européen Georges-Pompidou, Assistance publique-Hôpitaux de Paris, 20, rue Leblanc, F-75015 Paris, France

e-mail : alexandre.karras@aphp.fr

Faculté de médecine, université Paris-Descartes, 15 rue de l'École de médecine, F-75270 Paris Cedex 06, France
}

\begin{abstract}
Hemophagocytic lymphohistiocytosis (HLH) is a rare condition that complicates some rare inherited forms of immune deficiency, or occurring during the course of haematological (mainly non-Hodgkin lymphomas), infectious (herperviruses, bacteria or parasitosis) or autoimmune diseases (lupus, Still's disease). It is defined by an acute overactivation of the immune system, involving particularly the macrophages and the cytotoxic T-cells. HLH induces a massive release of proinflammatory cytokines that generate fever, cytopenias, organomegaly, hepatopathy and coagulopathy. A multi-organ failure is usual in this situation, requiring admission in intensive care unit, with an overall mortality rate above $50 \%$ in many series. Acute renal failure is frequently observed among HLH patients, due to acute tubular necrosis, tubulo-interstitial inflammatory nephritis or specific glomerulopathy with nephrotic syndrome. Identification of this disease is a real challenge for the intensivist, especially in patients presenting like sepsis or septic shock, but positive diagnosis can modify therapeutic management by leading to initiation of chemotherapy or immunosuppressive drugs, according to the specific aetiology of HLH.
\end{abstract}

Keywords Hemophagocytic lymphohistiocytosis · Macrophage activation syndrome $\cdot$ Acute kidney injury

\section{Introduction}

Le syndrome d'activation macrophagique (SAM) ou hémophagocytose lymphohistiocytaire (HLH) est une pathologie décrite initialement dans les années 1950, mais individualisée depuis la description des hémophagocytoses postvirales par Risdall et al. en 1979 [1]. Le SAM peut être observé dans des situations cliniques variées, rencontrées en médecine interne (infectiologie, hématologie et cancérologie, maladies systémiques) ainsi qu'en réanimation [2-8]. Il se traduit par un ensemble de signes cliniques, biologiques et histologiques 
non spécifiques, mais dont l'association doit faire évoquer le diagnostic. Son pronostic est sévère et le traitement encore mal codifié à ce jour.

\section{Syndrome d'activation macrophagique}

\section{Épidémiologie}

Le SAM est une pathologie dont la prévalence est probablement sous-estimée. Pour les formes pédiatriques, l'incidence annuelle a été estimée à 1,2 cas/million $[9,10]$, mais les formes de l'adulte, secondaires à des pathologiques infectieuses ou néoplasiques, sont probablement beaucoup plus fréquentes. Lorsqu'on étudie la prévalence de l'hémophagocytose (pas toujours associée à un réel SAM), à partir de séries se fondant sur des prélèvements médullaires, celle-ci est estimée aux environs de $1 \%$.

\section{Clinique}

Le tableau clinique est souvent assez brutal, associant de façon fréquente [11] :

- une fièvre, constante et importante, avec altération souvent marquée de l'état général ;

- une hépatosplénomégalie présente dans 40 à $70 \%$ des cas ;

- des adénopathies périphériques dans 30 à $70 \%$ des cas ;

- une éruption cutanée morbilliforme dans 10 à $20 \%$ des cas ;

- des signes digestifs inconstants (nausées, vomissements, diarrhée, douleur abdominale) ;

- des signes neurologiques (convulsions, irritation méningée, signes de localisation) et pulmonaires (dyspnée et toux avec sur l'imagerie un infiltrat ou une pleurésie), rares, mais pouvant parfois dominer le tableau clinique, surtout chez l'enfant ;

- des signes de défaillance multiviscérale (hémorragie viscérale dans le cadre des coagulations intravasculaires disséminées [CIVD], ictère, collapsus et détresse respiratoire).

\section{Biologie}

Les anomalies biologiques sont nombreuses, souvent majeures, mais non spécifiques. C'est leur association qui amène à évoquer le diagnostic de SAM :

- bi- ou pancytopénie périphérique constante, souvent profonde. La thrombopénie est l'anomalie la plus précoce et la plus fréquente, de mécanisme central, mais aussi parfois périphérique, par CIVD. L'anémie est fréquemment profonde et de constitution rapide, arégénérative, avec stigmates d'anémie hémolytique intratissulaire. La leucopénie est plus inconstante et tardive, portant surtout sur les polynucléaires neutrophiles ;

- troubles de l'hémostase retrouvés dans 50 à $70 \%$ des cas. On note essentiellement une hypofibrinémie soit isolée, soit associée à l'allongement des temps de thrombine, de prothrombine et de céphaline activée, témoignant d'une activation de la coagulation, voire d'une réelle CIVD, ce qui constitue un facteur de mauvais pronostic, avec la survenue de complications hémorragiques pouvant être fatales ;

- atteinte hépatique biologique retrouvée dans $40 \%$ des cas, se traduisant habituellement par une cytolyse parfois massive, pouvant évoluer vers l'insuffisance hépatocellulaire. La cholestase, souvent plus tardive, est corrélée à un pronostic plus défavorable ;

- augmentation constante des LDH plasmatiques, reflet de la lyse cellulaire ;

- hypertriglycéridémie précoce, pouvant atteindre des taux à plus de dix fois la normale ;

- augmentation parfois spectaculaire du taux de ferritine plasmatique, multifactorielle (syndrome inflammatoire, nécrose hépatocellulaire) ;

- hyponatrémie fréquente par probable sécrétion inappropriée d'hormone antidiurétique (ADH).

\section{Cytologie et histologie}

La cytologie médullaire permet le plus souvent d'affirmer le diagnostic quand elle est associée à des signes cliniques et biologiques évocateurs de SAM. Le myélogramme retrouve une moelle riche, avec une infiltration médullaire par des histiocytes d'aspect cytologique bénin, ce qui les différencie des histiocytoses malignes. Le pourcentage de macrophages est, pour certains auteurs, un critère diagnostique important (ils doivent représenter plus de $3 \%$ des cellules nucléées pour Tsuda [6]), mais aucune étude ne retrouve de relation entre le nombre d'histiocytes médullaires et la gravité de la maladie. Ces macrophages médullaires présentent de nombreuses vacuoles intracytoplasmiques, contenant des éléments cellulaires sanguins (érythrocytes, érythroblastes, granulocytes, plaquettes, lymphocytes) ou leurs précurseurs hématopoiétiques intacts ou partiellement digérés. Une érythroblastose est fréquente, témoin de l'érythropoï̀se réactionnelle à l'hémolyse intramédullaire.

D'autres examens cytologiques (cytoponction ganglionnaire, liquide d'ascite, liquide céphalorachidien) sont parfois informatifs, révélant des images d'hémophagocytose extramédullaire. Les biopsies ostéomédullaire mais également ganglionnaire ou hépatique [12] peuvent souvent confirmer l'hémophagocytose dans les organes hématopoïtiques, avec prolifération de macrophages dans les logettes osseuses, les 
sinus corticaux des ganglions, les sinusoïdes hépatiques ou les cordons de la pulpe rouge splénique. Plus rarement, l'hémophagocytose peut être mise en évidence dans d'autres organes, tels la peau ou le poumon, voire parfois les reins. En dehors du rôle important de ces examens histologiques pour confirmer le diagnostic de SAM, ils contribuent au bilan étiologique du SAM, permettant d'affirmer ou d'écarter une hémopathie maligne sous-jacente.

\section{Physiopathologie}

L'étiologie du SAM reste encore obscure, mais les progrès récents dans l'étude génétique des formes familiales apportent quelques éléments essentiels dans sa compréhension. Pour la plupart des auteurs, le déclenchement de cette pathologie est lié à une activation anormale des lymphocytes $\mathrm{T}$, favorisée par une infection ou un déficit congénital des mécanismes immunomodulateurs. Ces lymphocytes $\mathrm{T}$, essentiellement de profil Th1, produisent de grandes quantités de cytokines pro-inflammatoires qui stimulent la réponse macrophagique. Le défaut de cytotoxicité qui est observé, notamment dans les formes familiales, joue un rôle important, induisant une diminution de la régulation des cellules dendritiques et plus généralement des cellules présentatrices d'antigènes, qui ne sont plus lysées, mais qui continuent à activer les lymphocytes $\mathrm{T}$ [13]. La production de cytokines par les macrophages activés exerce a contrario un rétrocontrôle positif sur les lymphocytes $T$, entretenant une suractivation délétère du système immunitaire. L'activation lymphocytaire $\mathrm{T}$ se reflète dans l'augmentation des taux plasmatiques de récepteur soluble de l'IL-2 (IL : interleukine) [sIL-2R] ainsi que d'interféron gamma (IFN- $\gamma$ ) circulant, corrélés à la gravité de la maladie et au pronostic de l'affection. À l'inverse, les taux plasmatiques d'IL-4 sont effondrés dans ce contexte, avec un déséquilibre de la balance Th1/Th2 au profit des lymphocytes Th1. Les lymphocytes CD8+ sont ainsi dans un état d'activation excessive, avec élévation des taux sanguins de CD8 soluble et de ligand soluble de Fas (sFasL). L'activation des macrophages est responsable à la fois du syndrome inflammatoire et de la fièvre (production d'IL-1, de tumor necrosis factor- $\alpha$ [TNF- $\alpha]$ et d'IL-6), de la pancytopénie par phagocytose des éléments figurés du sang et de l'amplification de la réponse lymphocytaire par production d'IL-12, d'IL-1 et de TNF- $\alpha$. La pancytopénie serait également favorisée par l'action myélosuppressive de l'IFN- $\gamma$ (régulation positive de l'expression de Fas sur les cellules hématopoïétiques $\mathrm{CD} 34+$, ce qui les rend sensibles à l'action cytotoxique du FasL). L'organomégalie est liée à l'infiltration tissulaire par des macrophages activés. Les perturbations du bilan hépatique sont la conséquence à la fois de l'activation macrophagique intrahépatique (cellules de Kupffer), ainsi que de l'induction par l'IFN- $\gamma$, de la molécule Fas sur les hépatocytes. L'hypertri- glycéridémie, classique dans le SAM, est liée à l'inhibition de la lipoprotéine lipase par l'association TNF- $\alpha$ et IL-1. L'hyperferritinémie résulterait de l'érythrophagocytose, du dysfonctionnement hépatique, mais surtout de l'inflammation spécifique, avec stimulation de la production d'hepcidine.

\section{Étiologie}

Les causes de SAM peuvent être séparées en formes primitives, souvent familiales, liées à des anomalies génétiques et touchant essentiellement l'enfant, et en formes secondaires, avec des cas sporadiques d'hémophagocytose réactionnelle, compliquant une maladie infectieuse, néoplasique ou autoimmune.

\section{HLH génétiques ou FLH}

Plusieurs pathologies héréditaires du système immunitaire peuvent se compliquer d'une HLH, au cours d'une phase accélérée de la maladie, souvent déclenchée par un stimulus infectieux. Certaines sont désormais bien individualisées et les anomalies génétiques à leur origine clairement déterminées [14]:

- la lymphohistiocytose familiale (familial lymphohistiocytosis) de type 2 ou FLH2 (maladie la plus fréquente parmi ces pathologies) est une maladie de la première enfance (âge moyen des patients : deux mois), transmise sur le mode autosomique récessif. Au décours d'une infection, souvent virale, les organes lymphoïdes sont infiltrés par une population cellulaire polyclonale mixte, lymphocytaire $\mathrm{T}$ et macrophagique, avec une évolution secondaire quasi constante vers le décès en l'absence de traitement. Il existe souvent des antécédents familiaux (50\% dans le registre international) ou une consanguinité $(10 \%$ des cas). Cette pathologie est secondaire à une mutation inactivatrice du gène de la perforine $(P R F 1)$ [15], conduisant à un défaut de cytotoxicité des lymphocytes CD8+, empêchant la lyse des cellules présentatrices d'antigènes exprimant à leur surface un antigène viral ou bactérien et, par conséquent, entretenant une activation permanente d'une population lymphocytaire dirigée vis-à-vis de cet antigène ;

- les FLH de type 3, 4 ou 5 sont secondaires à des mutations portant sur d'autres gènes (respectivement UNC13D, STX11, STXBP2), codant pour des protéines (Munc13-4, syntaxine 11, Munc18-2) impliquées dans le trafficking et la fusion des membranes cellulaires. Le gène responsable de FLH de type 1 reste encore indéterminé à ce jour ;

- le syndrome de Chediak-Higashi et le syndrome de Griscelli sont caractérisés par un albinisme partiel, cutané et oculaire associé à un déficit immunitaire $\mathrm{T}$ cytotoxique et 
$\mathrm{NK}$, avec présence de grandes granulations intracytoplasmiques. Les gènes mutés dans cette maladie codent pour les protéines LYST (LYSosomal Trafficking regulator) [16] et RAB27A [17], impliquées dans l'adressage des protéines intracellulaires vers les vésicules d'exocytose ou leur fusion avec la membrane cellulaire ;

- le syndrome de Purtilo (X-linked lymphoproliferative syndrome) est un déficit immunitaire héréditaire rare $\left(1 / 10^{6}\right.$ garçons), caractérisé par une susceptibilité accrue à l'infection par le virus d'Epstein-Barr (EBV), conduisant à la survenue d'une mononucléose infectieuse gravissime avec HLH. Il est secondaire à une mutation portant sur un gène du chromosome $X$, codant pour une protéine nommée SH2D1A, exprimée dans les lymphocytes T et impliquée dans le contrôle de la réponse lymphocytaire.

Toutes ces pathologies ont en commun une activation primitive lymphocytaire $\mathrm{T}$, souvent déclenchée par une infection opportuniste, avec production majeure de cytokines proinflammatoires (IFN- $\gamma$, IL-1, IL-6, TNF- $\alpha$ ), puis une activation macrophagique qui participe aux lésions tissulaires des organes cibles.

\section{SAM réactionnels}

L'activation macrophagique peut apparaître au cours de diverses pathologies, infectieuses, néoplasiques ou auto-immunes. Le diagnostic étiologique demeure souvent négatif, car le spectre des pathologies associées à ce syndrome est extrêmement large, et le tableau clinique est habituellement dominé par les manifestations secondaires au SAM, occultant les signes spécifiques de la pathologie causale. Dans la revue de la littérature médicale publiée en 2014 par Ramos-Casals et al., analysant 2197 cas de SAM de l'adulte, on retrouve un facteur infectieux dans $50,4 \%$ des cas, une néoplasie dans $47,6 \%$, une maladie auto-immune dans $12,5 \%$ [11].

\section{- SAM postinfectieux}

Quasiment toutes les infections, bactériennes, virales, fungiques ou parasitaires, ont été associées au SAM [18], le plus souvent à titre anecdotique (Tableau 1). L'analyse des principales séries publiées révèle que les herpèsvirus (CMV, EBV, VZV, HSV, HHV-6) sont responsables de près de la moitié des cas de SAM postinfectieux, avec une nette prédominance de l'EBV. Suivent par ordre décroissant les mycobactéries, les bactéries intracellulaires et pyogènes, puis les parasites. Il n'est pas rare de retrouver deux agents infectieux pathogènes chez le même patient au cours d'une hémophagocytose. Il faut souligner qu'il est parfois difficile d'imputer la survenue d'un syndrome hémophagocytaire à une infection. D'une part, l'agent infectieux pourrait juste jouer le rôle de facteur déclenchant sur un terrain immuno-
Tableau 1 Critères diagnostiques HLH-2004 pour le syndrome d'activation macrophagique

a. Diagnostic moléculaire de FHL avec mise en évidence d'une mutation pathogène des gènes $P R F 1, U N C 13 D$, MUNC18-2, RAB27a, STX11, SH2D1A ou BIRC4 (recherche demandée dans les cas pédiatriques ou familiaux) ou

b. Présence d'au moins 5 parmi les 8 critères suivants :

1. Fièvre $>38,5{ }^{\circ} \mathrm{C}$

2. Splénomégalie

3. Cytopénie sur 2 ou 3 lignées $(\mathrm{Hb}<9 \mathrm{~g} / \mathrm{dl}$, polynucléaires neutrophiles $<10 \mathrm{G} / 1$, plaquettes $<100 \mathrm{G} / \mathrm{l}$ )

4. Hypertriglycéridémie $>2 \mathrm{mmol} / 1$ et/

ou hypofibrinogénémie $<1,5 \mathrm{~g} / 1$

5. Hémophagocytose (médullaire, splénique, hépatique ou ganglionnaire)

6. Diminution ou absence d'activité NK

7. Ferritinémie $>500 \mathrm{ng} / \mathrm{ml}$

8. Concentration de CD25 soluble (sIL2R) $>2400 \mathrm{U} / \mathrm{ml}$

logique particulier. D'autre part, l'immunosuppression qui résulte du SAM (secondaire à la leucopénie et parfois aux traitements administrés) peut favoriser les surinfections secondaires, venant parfois rendre impossible le diagnostic étiologique. Un grand nombre des cas rapportés dans la littérature surviennent sur un terrain d'immunodépression sous-jacente (infection à VIH, traitement immunosuppresseur pour une transplantation ou une maladie systémique, chimiothérapie anticancéreuse) $[19,20]$. Il est, de ce fait, difficile de savoir si l'immunodépression favorise l'activation macrophagique en soi ou tout simplement l'infection qui est à l'origine du SAM.

Les infections bactériennes sévères semblent également pouvoir évoluer vers un SAM, prenant, dans ce contexte, la forme d'une défaillance multiviscérale. Des bactéries communes (Escherichia coli, pneumocoque, autres bacilles Gram négatif, staphylocoques) ont été décrites à l'origine de réels syndromes hémophagocytaires. Une étude prospective en réanimation a montré que le myélogramme systématique chez des patients thrombopéniques au cours d'un choc septique montrait une hémophagocytose dans $60 \%$ des cas [21]. Cela démontre que l'hémophagocytose est probablement sous-estimée, surtout au cours des syndromes septiques, et qu'elle pourrait expliquer en partie les pancytopénies observées dans ce cadre nosologique.

- SAM associés à une néoplasie

L'hémophagocytose peut s'associer, compliquer ou même révéler une néoplasie évolutive, dans environ 30 à $50 \%$ des cas. La pathologie tumorale la plus fréquente est le 
lymphome de haut grade de malignité, essentiellement non hodgkinien de phénotype T ou NK. Ainsi dans la métaanalyse de Ramos-Casals et al. [11], les lymphomes représentent $76 \%$ des SAM associés à une pathologie tumorale, dont $35 \%$ de lymphomes T, $32 \%$ de lymphomes B, $6 \%$ de Hodgkin. Dans la série de Su et al. [22], sur les 23 patients atteints de lymphome avec activation macrophagique, 15 présentaient des lymphomes T associés à l'EBV. Cette entité hématologique, désormais bien reconnue, est marquée par une hémophagocytose fréquente et extrêmement sévère, puisque sur les 22 lymphomes T-EBV induits, rapportés par Yao et al. [23], 15 patients sont décédés d'un SAM d'évolution fulminante. À noter d'ailleurs qu'une publication récente [24] semble indiquer que la survenue fréquente d'un SAM au cours des lymphomes T/NK serait liée à une mutation somatique du gène ECSIT (Evolutionarily conserved signaling intermediate in Toll pathway»), impliqué dans la signalisation des récepteurs Toll, aboutissant à une suractivation de la voie NF-kB (nuclear factor-kappa B). Dans les lymphomes B, la fréquence du SAM est bien moindre : sept cas de SAM sur 105 lymphomes B dans la série de Miyahara et al. [25]. D'ailleurs, dans la série récente rapportée par Vick et al. [26], les lymphomes T ou NK représentent $46 \%$ des SAM néoplasiques versus $28 \%$ pour les lymphomes B. Les autres maladies néoplasiques associées au SAM peuvent être des hémopathies diverses (leucémies aiguës myéloblastique ou lymphoblastique, myélome multiple, myélodysplasie, syndromes myéloprolifératifs) ou des tumeurs solides variées (carcinome gastrique ou colique, thymome, sarcomes divers, cancer bronchopulmonaire à petites cellules, tumeurs germinales, etc.).

- SAM associés à une maladie systémique

L'hémophagocytose a été décrite au cours de certaines pathologies auto-immunes ou inflammatoires chroniques $[27,28]$. Plusieurs cas de lupus érythémateux systémique avec hémophagocytose sont signalés dans la littérature, avec une fréquence de $2,4 \%$ sur la série de Wong et al. [29], comprenant 250 patients lupiques suivis pendant 3,5 ans en moyenne. Les autres pathologies sont multiples : connectivite mixte, sclérodermie, maladie de Still, maladie de WeberChristian, maladie de Horton, polyarthrite rhumatoïde, entéropathies inflammatoires, maladie de Kikuchi. On retrouve ainsi une notion de maladie systémique chez $6-10 \%$ des malades ayant une hémophagocytose. Il est cependant souvent difficile de préciser si l'activation macrophagique est secondaire à la maladie auto-immune ou à une infection survenant chez des patients recevant souvent un ou des immunosuppresseur(s) au long cours.

De façon intéressante, certaines études récentes ont suggéré que certains cas de SAM réactionnels de l'adulte pouvaient survenir chez des patients présentant une prédisposi- tion génétique [30]. Ainsi, dans une série de 175 patients adultes [31], des mutations hypomorphes de PRF1, de MUNC13-4 ou de STXBP2 ont été retrouvées dans $14 \%$ des cas, suggérant que le stimulus néoplasique ou infectieux n'est parfois que le simple facteur déclenchant d'un syndrome hémophagocytaire d'origine génétique.

\section{Critères diagnostiques}

L'existence d'hémophagocytose sur les prélèvements cytologiques ou histologiques n'est ni suffisante ni même obligatoire pour porter le diagnostic de SAM. En effet, des images d'hémophagocytose sont rencontrées dans un certain nombre de situations cliniques, sans nécessairement être associées au cortège de signes cliniques et biologiques qui font la gravité du SAM. A contrario, les prélèvements tissulaires ne retrouvent pas toujours les images typiques, alors même que le tableau clinicobiologique est typique.

Les critères utilisés majoritairement, par la plupart des auteurs, sont ceux proposés par l'HLH-2004 [32] (Tableau 1).

Un score a été proposé pour valider plus spécifiquement le diagnostic de syndrome hémophagocytaire réactionnel, en se fondant sur neuf variables (immunosuppression sousjacente, fièvre, organomégalie, taux de triglycérides, ferritine, SGOT, fibrinogène, cytopénies, hémophagocytose médullaire) [33].

\section{Traitement}

Le traitement des syndromes hémophagocytaires est assez mal codifié, et aucune étude thérapeutique n'a été réalisée, en dehors du cadre des hémophagocytoses héréditaires de l'enfant [34]. On ne parlera pas ici des traitements symptomatiques, qui consistent à corriger les troubles hydroélectrolytiques (fréquents dans cette pathologie) et à réaliser des transfusions itératives, nécessaires devant des pancytopénies souvent très profondes.

Dans les FLH, le traitement a beaucoup évolué ces dernières décennies, permettant une amélioration notable du pronostic. L'utilisation d'étoposide (VP16) avait permis depuis les années 1980 d'obtenir des rémissions temporaires de la maladie, cet agent cytotoxique étant toujours considéré comme un des éléments essentiels pour le contrôle de la prolifération et de la suractivation des histiocytes/macrophages. Les injections intrathécales de méthotrexate ont par ailleurs permis de traiter les atteintes du système nerveux central (SNC) fréquentes et très péjoratives dans ces formes pédiatriques de SAM (le VP16 ne passant pas la barrière hématoméningée). La compréhension de la physiopathologie, mettant en cause l'activation lymphocytaire $\mathrm{T}$ dans la stimulation des macrophages, avait également conduit à proposer un traitement par anticorps antilymphocytaire, puis ciclosporine. Le protocole thérapeutique communément 
admis désormais (HLH-2004) comporte l'association de dexaméthasone, de ciclosporine, d'étoposide (avec injections intrathécales de méthotrexate si atteinte du SNC), suivie, dès la rémission obtenue, d'une greffe de moelle allogénique, seul traitement permettant de traiter définitivement la HLH d'origine génétique [35].

Ces différents schémas thérapeutiques ont été transposés, avec plus ou moins de succès, dans les hémophagocytoses réactionnelles [36]. Le traitement de la cause est évidemment essentiel dans ce cas, avec mise en route rapide d'un traitement anti-infectieux en cas de SAM postinfectieux ou d'une chimiothérapie dans les hémopathies malignes associée à une hémophagocytose. Quant aux manifestations liées à l'hémophagocytose, elles ont conduit plusieurs équipes à tester l'utilité de l'étoposide [37] et des stéroïdes (notamment dans les hémopathies ou les infections à EBV [38]), voire de la ciclosporine en cas de pathologie auto-immune associée. Les immunoglobulines polyvalentes (IgIV) ont également été proposées avec succès dans les hémophagocytoses postvirales [39], leur mécanisme d'action restant difficile à déterminer (action anti-infectieuse directe, immunomodulation, etc.). Des cas de réponses à des biothérapies ciblées ont été rapportés, notamment avec des anti-TNF (infliximab, étanercept), l'anakinra (anti-IL1R) ou le tocilizumab (anti-IL6) dans le cadre de maladies auto-immunes associées à un SAM réactionnel. Malheureusement, aucune étude prospective contrôlée n'a été mise en œuvre à ce jour pour prouver l'efficacité de ce traitement empirique.

Le schéma proposé actuellement dans la prise en charge d'une hémophagocytose secondaire à une infection où une maladie systémique place en première ligne les IgIV associées à un traitement anti-infectieux à large spectre (antibactérien, antiviral et antifungique). Les corticoïdes sont proposés surtout dans un contexte évident de pathologie autoimmune, mais leur utilisation systématique est freinée par la crainte de favoriser une infection sous-jacente non maîtrisée. Le VP16, dont l'utilisation répétée peut conduire à une myélodysplasie et à une leucémie secondaire, est réservé aux lymphomes avec SAM ou en cas d'échec des autres traitements [26,34]. Il faut d'ailleurs garder en tête que les doses de ce traitement, éliminées par voie rénale et hépatique, doivent être diminuées de façon drastique en cas d'insuffisance rénale ou de cholestase majeure. Les traitements d'avenir sont peut-être les biothérapies ciblant spécifiquement les cytokines qui sont au centre de cette « tempête » cytokinique.

\section{Pronostic}

Dans les SAM héréditaires, la greffe de moelle allogénique a totalement modifié le pronostic. Ainsi selon le Registre international de lymphohistiocytose familiale (122 patients), la survie à cinq ans est à peine de $10 \%$ pour les patients traités par chimiothérapie conventionnelle (VP16-corticoïdesméthotrexate intrathécal), alors qu'elle est de plus de $65 \%$ pour les patients ayant pu bénéficier d'une allogreffe $[35,40]$. Dans les SAM réactionnels, le pronostic dépend de plusieurs paramètres : précocité du diagnostic, positivité du bilan étiologique, mise en route précoce d'un traitement anti-infectieux adapté, étiologie néoplasique associée, statut immunitaire antérieur (VIH, immunodéprimé). Dans la méta-analyse de Ramos-Casals et al. [11], le pronostic est défavorable dans $41 \%$ des cas, montrant bien la gravité de cette pathologie, notamment dans les SAM associés à un lymphome, où la mortalité atteint jusqu'à $90 \%$ selon les séries [41,42]. Dans l'analyse rétrospective japonaise de 134 cas de lymphome avec activation macrophagique, la médiane de survie est de 69 jours pour les lymphomes $\mathrm{T}$ et de 242 jours pour les lymphomes B.

L'analyse de quelques grandes séries permet cependant de dégager certains autres facteurs de mauvais pronostic, indépendamment de l'étiologie, notamment l'âge supérieur à 60 ans, la ferritinémie supérieure à $50000 \mu \mathrm{g} / 1$, l'hyperbilirubinémie supérieure à $34 \mu \mathrm{mol} / 1$, la thrombopénie inférieure à $80 \mathrm{G} / 1$, la neutropénie inférieure à $0,5 \mathrm{G} / 1$ [26].

\section{Atteinte rénale dans le syndrome d'activation macrophagique}

L'atteinte rénale du SAM est assez mal décrite dans la plupart des grandes séries rétrospectives. Néanmoins, l'insuffisance rénale aiguë est une complication fréquente, observée dans 15 à $60 \%$ des cas de SAM, en fonction du type de patients et de la définition retenue au plan néphrologique $[2,43,44]$. Cette insuffisance rénale aiguë est classiquement attribuée à une nécrose tubulaire aiguë (NTA) faisant suite à la défaillance multiviscérale du SAM ou aux traitements néphrotoxiques utilisés dans ce contexte [45], même si les données étayées par des biopsies rénales sont rares. Dans une petite série autopsique [46], une NTA était notée dans $45 \%$ des cas de SAM, une néphropathie interstitielle aiguë dans $55 \%$ des reins analysés. Dans la série d'une équipe francilienne, reprenant 95 patients adultes admis en réanimation pour un SAM réactionnel (hémopathie retrouvée dans $77 \%$ des cas), une insuffisance rénale aiguë selon les critères KDIGO était retrouvée dans $62 \%$ des cas [44]. Plus de la moitié $(59 \%)$ de ces insuffisances rénales aiguës étaient sévères, nécessitant l'épuration extrarénale, alors que les causes retenues à l'origine de la néphropathie étaient une insuffisance rénale fonctionnelle ( $46 \%$ des cas), une NTA (49\%), un syndrome de lyse tumorale (29\%), l'utilisation d'un médicament néphrotoxique $(70 \%)$ ou une glomérulopathie $(17 \%)$. Une biopsie rénale était assez rarement réalisée dans cette série, et la majorité de ces diagnostics étaient des diagnostics de présomption fondés sur le contexte 
clinique et les données biologiques disponibles. Il faut par ailleurs souligner le fait que cette étude rapporte des patients globalement plus sévères que ceux souffrant de SAM toutvenant, avec nécessité de ventilation mécanique dans $46 \%$ des cas, d'utilisation d'amines vasoactives dans $42 \%$ des cas. La mortalité globale dans cette série était de $56 \%$ à six mois. Parmi les 22 patients avec insuffisance rénale aiguë survivant plus de six mois, $32 \%$ gardaient une dysfonction rénale chronique résiduelle.

Parmi les causes les plus rares d'insuffisance rénale survenant dans ce contexte, d'exceptionnels cas d'hémophagocytose intrarénale ont été décrits [47], ainsi que des cas d'infiltration lymphomateuse interstitielle ou rétropéritonéale, dans les SAM associés aux hémopathies. L'existence d'un glomérulopathie au cours d'un SAM a également été rapportée [48], et il est intéressant de noter que dans la série d'Aulagnon et al., la protéinurie médiane était de $1,7 \mathrm{mg} / \mathrm{g}$ de créatininurie, avec un réel syndrome néphrotique dans certains cas. Une série rétrospective multicentrique a décrit neuf cas de syndrome néphrotique associé à un SAM, avec une documentation histologique rénale par ponction-biopsie rénale ou examen autopsique [49]. L'étiologie du SAM était hématologique dans deux tiers des cas, dont quatre cas de lymphome $\mathrm{T}$. Le syndrome néphrotique était contemporain du SAM dans la majorité des cas, avec une protéinurie moyenne de $11 \mathrm{~g} / 24 \mathrm{~h}$, alors que la valeur minimale de l'albuminémie était de $17 \mathrm{~g} / \mathrm{l}$. L'insuffisance rénale était constante, avec nécessité de dialyse dans plus de la moitié des cas. Le type de glomérulopathie la plus fréquemment rencontrée $(n=5)$ était la collapsing glomerulopathy $(\mathrm{CG})$, forme de hyalinose segmentaire et focale s'accompagnant d'un collapsus glomérulaire, alors que le diagnostic de néphropathie à lésions glomérulaires minimes (NLGM) était retenu dans les autres cas. À noter que quelques cas de microangiopathie thrombotique ont été rapportés au cours des syndromes hémophagocytaires, pouvant parfois expliquer la dysfonction rénale [50].

\section{Discussion et conclusion}

La survenue d'une dysfonction multiviscérale au cours d'une HLH, génétique ou réactionnelle, est désormais bien reconnue et doit être détectée précocement pour améliorer la prise en charge symptomatique de ces patients, le temps que l'étiologie soit identifiée et le traitement spécifique initié, comportant un immunosuppresseur, une chimiothérapie, un traitement anti-infectieux, voire une combinaison de ces stratégies thérapeutiques. L'atteinte rénale, souvent au second plan chez ces patients présentant une instabilité hémodynamique, une défaillance respiratoire, une atteinte hépatique ou une coagulopathie sévère, est un réel facteur de mauvais pronostic, tant sur le plan vital que fonctionnel.
La question qui demeure ouverte néanmoins concerne l'existence ou non d'un SAM au cours des sepsis. François et al. avaient rapporté en 1997 une série de 50 patients présentant un sepsis et une thrombocytopénie, explorés par myélogramme, et présentant une hémophagocytose pour 32 d'entre eux (soit $64 \%$ ). Ainsi, une série autopsique allemande de 107 patients décédés en unité de soins intensifs a retrouvé une hémophagocytose dans $64 \%$ des cas [51], alors qu'une équipe française a récemment rapporté son expérience de patients ayant " bénéficié » en réanimation d'un myélogramme pour cytopénie, retrouvant une hémophagocytose médullaire dans $106 / 460$ cas [52]. À noter que la grande majorité de ces patients présentaient un sepsis bactérien, avec bicytopénie dans $53 \%$ des cas et pancytopénie dans $17 \%$, une ferritinémie médiane à $1110 \mu \mathrm{g} / \mathrm{l}$ et une triglycéridémie médiane à $2,29 \mathrm{mmol} / \mathrm{l}$. Toute la difficulté dans ce contexte est de différencier hémophagocytose " physiologique » associée au sepsis, relevant juste de la prise en charge anti-infectieuse usuelle, HLH réactionnelle sévère, justifiant la prescription d'une corticothérapie, d'un immunosuppresseur, voire d'une chimiothérapie, mais aussi FLH d'origine génétique nécessitant une prise en charge spécifique avec greffe de moelle. Diverses propositions ont été faites pour aider le clinicien à prendre une telle décision [33,53], mais ces scores diagnostiques n'ont pas encore été validés dans les différentes populations en cause, pédiatriques, hématologiques ou réanimatoires. Des études à plus grande échelle sont nécessaires pour affiner la description clinique et biologique de chacune de ces formes faisant partie du spectre de l'hémophagocytose systémique et identifier les biomarqueurs qui permettront d'améliorer la prise en charge des patients.

Liens d'intérêts : l'auteur déclare ne pas avoir de lien d'intérêt.

\section{Références}

1. Risdall RJ, McKenna RW, Nesbit ME, Krivit W, Balfour HH Jr, Simmons RL, Brunning RD, (1979) Virus-associated hemophagocytic syndrome: a benign histiocytic proliferation distinct from malignant histiocytosis. Cancer 44: 993-1002

2. Reiner AP, Spivak JL, (1988) Hematophagic histiocytosis. A report of 23 new patients and a review of the literature. Medicine 67: 369-388

3. Risdall RJ, Brunning RD, Hernandez JI, Gordon DH, (1984) Bacteria-associated hemophagocytic syndrome. Cancer 54: 2968-2972

4. Wong KF, Chan JK, (1992) Reactive hemophagocytic syndrome: a clinicopathologic study of 40 patients in an Oriental population. Am J Med 93: 177-180

5. Sailler L, Duchayne E, Marchou B, Brousset P, Pris J, Massip P, Corberand J, Arlet P, (1997) Etiological aspects of reactive hemophagocytoses: retrospective study in 99 patients. Rev Med Interne 18: 855-864 
6. Tsuda H, (1997) Hemophagocytic syndrome (HPS) in children and adults. Int J Hematol 65: 215-226

7. Kaito K, Kobayashi M, Katayama T, Otsubo H, Ogasawara Y, Sekita T, Saeki A, Sakamoto M, Nishiwaki K, Masuoka H, Shimada T, Yoshida M, Hosoya T, (1997) Prognostic factors of hemophagocytic syndrome in adults: analysis of 34 cases. Eur J Haematol 59: 247-253

8. Rostaing L, Fillola G, Baron E, Cisterne JM, Durand D, (1995) Course of hemophagocytic histiocytic syndrome in renal transplant patients. Transplantation 60: 506-509

9. Ishii E, Ohga S, Imashuku S, Yasukawa M, Tsuda H, Miura I, Yamamoto K, Horiuchi H, Takada K, Ohshima K, Nakamura S, Kinukawa N, Oshimi K, Kawa K, (2007) Nationwide survey of hemophagocytic lymphohistiocytosis in Japan. Int J Hematol 86: $58-65$

10. Aricò M, Danesino C, Pende D, Moretta L, (2001) Pathogenesis of haemophagocytic lymphohistiocytosis. Br J Haematol 114: 761-769

11. Ramos-Casals M, Brito-Zerón P, López-Guillermo A, Khamashta MA, Bosch X, (2014) Adult haemophagocytic syndrome. Lancet 383: $1503-1516$

12. de Kerguenec C, Hillaire S, Molinié V, Gardin C, Degott C, Erlinger S, Valla D, (2001) Hepatic manifestations of hemophagocytic syndrome: a study of 30 cases. Am J Gastroenterol 96: 852-857

13. Terrell CE, Jordan MB, (2013) Perforin deficiency impairs a critical immunoregulatory loop involving murine CD8+ T-cells and dendritic cells. Blood 121: 5184-5191

14. Dufourcq-Lagelouse R, Pastural E, Barrat FJ, Feldmann J, Le Deist F, Fischer A, De Saint Basile G, (1999) Genetic basis of hemophagocytic lymphohistiocytosis syndrome (Review). Int J Mol Med 4: 127-133

15. Stepp SE, Dufourcq-Lagelouse R, Le Deist F, Bhawan S, Certain S, Mathew PA, Henter JI, Bennett M, Fischer A, de Saint Basile G, Kumar V, (1999) Perforin gene defects in familial hemophagocytic lymphohistiocytosis. Science 286: 1957-1959

16. Barbosa MD, Barrat FJ, Tchernev VT, Nguyen QA, Mishra VS, Colman SD, Pastural E, Dufourcq-Lagelouse R, Fischer A, Holcombe RF, Wallace MR, Brandt SJ, de Saint Basile G, Kingsmore SF, (1997) Identification of mutations in two major mRNA isoforms of the Chediak-Higashi syndrome gene in human and mouse. Hum Mol Genet 6: 1091-1098

17. Ménasché G, Pastural E, Feldmann J, Certain S, Ersoy F, Dupuis S, Wulffraat N, Bianchi D, Fischer A, Le Deist F, de Saint Basile G, (2000) Mutations in RAB27A cause Griscelli syndrome associated with haemophagocytic syndrome. Nat Genet 25: 173-176

18. Fisman DN, (2000) Hemophagocytic syndromes and infection. Emerging Infect Dis 6: 601-608

19. Karras A, Thervet E, Legendre C ; Groupe coopératif de transplantation d'Île-de-France, (2004) Hemophagocytic syndrome in renal transplant recipients: report of 17 cases and review of literature. Transplantation 77: 238-243

20. Fardet L, Lambotte O, Meynard JL, Kamouh W, Galicier L, Marzac C, de Labarthe A, Cabane J, Lebbe C, Coppo P, Molina JM, Martinez V, (2010) Reactive haemophagocytic syndrome in 58 HIV-1-infected patients: clinical features, underlying diseases and prognosis. AIDS 24: 1299-1306

21. Stéphan F, Thiolière B, Verdy E, Tulliez M, (1997) Role of hemophagocytic histiocytosis in the etiology of thrombocytopenia in patients with sepsis syndrome or septic shock. Clin Infect Dis 25: 1159-1164

22. Su IJ, Wang CH, Cheng AL, Chen RL, (1995) Hemophagocytic syndrome in Epstein-Barr virus-associated T-lymphoproliferative disorders: disease spectrum, pathogenesis, and management. Leuk Lymphoma 19: 401-406
23. Yao M, Cheng AL, Su IJ, Lin MT, Uen WC, Tien HF, Wang CH, Chen YC, (1994) Clinicopathological spectrum of haemophagocytic syndrome in Epstein-Barr virus-associated peripheral T-cell lymphoma. Br J Haematol 87: 535-543

24. Wen H, Ma H, Cai Q, Lin S, Lei X, He B, Wu S, Wang Z, Gao Y, Liu W, Liu W, Tao Q, Long Z, Yan M, Li D, Kelley KW, Yang Y, Huang H, Liu Q, (2018) Recurrent ECSIT mutation encoding V140A triggers hyperinflammation and promotes hemophagocytic syndrome in extranodal NK/T cell lymphoma. Nat Med 24: 154-164

25. Miyahara M, Sano M, Shibata K, Matsuzaki M, Ibaraki K, Shimamoto Y, Tokunaga O, (2000) B-cell lymphoma-associated hemophagocytic syndrome: clinicopathological characteristics. Ann Hematol 79: 378-388.

26. Vick EJ, Patel K, Prouet P, Martin MG, (2017) Proliferation through activation: hemophagocytic lymphohistiocytosis in hematologic malignancy. Blood Adv 1: 779-791

27. Papo T, Andre MH, Amoura Z, Lortholary O, Tribout B, Guillevin L, Piette JC, (1999) The spectrum of reactive hemophagocytic syndrome in systemic lupus erythematosus. J Rheumatol 26: 927-930

28. Dhote R, Simon J, Papo T, Detournay B, Sailler L, Andre MH, Dupond JL, Larroche C, Piette AM, Mechenstock D, Ziza JM, Arlaud J, Labussiere AS, Desvaux A, Baty V, Blanche P, Schaeffer A, Piette JC, Guillevin L, Boissonnas A, Christoforov B, (2003) Reactive hemophagocytic syndrome in adult systemic disease: report of twenty-six cases and literature review. Arthritis Rheum 49: 633-639

29. Wong KF, Hui PK, Chan JK, Chan YW, Ha SY, (1991) The acute lupus hemophagocytic syndrome. Ann Intern Med 114: 387-390

30. Sieni E, Cetica V, Piccin A, Gherlinzoni F, Sasso FC, Rabusin M, Attard L, Bosi A, Pende D, Moretta L, Aricò M, (2012) Familial hemophagocytic lymphohistiocytosis may present during adulthood: clinical and genetic features of a small series. PLoS ONE 7: e44649

31. Zhang K, Jordan MB, Marsh RA, Johnson JA, Kissell D, Meller J, Villanueva J, Risma KA, Wei Q, Klein PS, Filipovich AH, (2011) Hypomorphic mutations in PRF1, MUNC13-4, and STXBP2 are associated with adult-onset familial HLH. Blood 118: 5794-5798

32. Henter JI, Horne A, Aricó M, Egeler RM, Filipovich AH, Imashuku S, Ladisch S, McClain K, Webb D, Winiarski J, Janka G, (2007) HLH-2004: diagnostic and therapeutic guidelines for hemophagocytic lymphohistiocytosis. Pediatr Blood Cancer 48: 124-131

33. Fardet L, Galicier L, Lambotte O, Marzac C, Aumont C, Chahwan D, Coppo P, Hejblum G, (2014) Development and validation of the HScore, a score for the diagnosis of reactive hemophagocytic syndrome. Arthritis Rheumatol 66: 2613-2620

34. Jordan MB, Allen CE, Weitzman S, Filipovich AH, McClain KL, (2011) How I treat hemophagocytic lymphohistiocytoses? Blood 118: 4041-4052

35. Bergsten E, Horne A, Aricó M, Astigarraga I, Egeler RM, Filipovich $\mathrm{AH}$, Ishii $\mathrm{E}$, Janka $\mathrm{G}$, Ladisch S, Lehmberg K, McClain KL, Minkov M, Montgomery S, Nanduri V, Rosso D, Henter JI, (2017) Confirmed efficacy of etoposide and dexamethasone in HLH treatment: long-term results of the cooperative HLH-2004 study. Blood 130: 2728-2738

36. Brisse E, Matthys P, Wouters CH, (2016) Understanding the spectrum of haemophagocytic lymphohistiocytosis: update on diagnostic challenges and therapeutic options. $\mathrm{Br} \mathrm{J}$ Haematol 174: $175-187$

37. Arca M, Fardet L, Galicier L, Rivière S, Marzac C, Aumont C, Lambotte O, Coppo P, (2015) Prognostic factors of early death in a cohort of 162 adult haemophagocytic syndrome: impact of triggering disease and early treatment with etoposide. $\mathrm{Br} \mathrm{J}$ Haematol 168: $63-68$ 
38. Imashuku S, Kuriyama K, Teramura T, Ishii E, Kinugawa N, Kato M, Sako M, Hibi S, (2001) Requirement for etoposide in the treatment of Epstein-Barr virus-associated hemophagocytic lymphohistiocytosis. J Clin Oncol 19: 2665-2673

39. Larroche C, Bruneel F, André MH, Bader-Meunier B, Baruchel A, Tribout B, Genereau T, Zunic P ; Comité d'évaluation et de diffusion des innovations technologiques (Cedit), (2000) Intravenously administered gamma-globulins in reactive hemaphagocytic syndrome. Multicenter study to assess their importance, by the immunoglobulins group of experts of CEDIT of the AP-HP. Ann Med Interne (Paris) 151: 533-539

40. Aricò M, Janka G, Fischer A, Henter JI, Blanche S, Elinder G, Martinetti M, Rusca MP, (1996) Hemophagocytic lymphohistiocytosis. Report of 122 children from the International Registry. FHL Study Group of the Histiocyte Society. Leukemia 10: 197-203

41. Majluf-Cruz A, Sosa-Camas R, Pérez-Ramírez O, Rosas-Cabral A, Vargas-Vorackova F, Labardini-Méndez J, (1998) Hemophagocytic syndrome associated with hematological neoplasias. Leuk Res 22: 893-898

42. Yu JT, Wang CY, Yang Y, Wang RC, Chang KH, Hwang WL, Teng CL, (2013) Lymphoma-associated hemophagocytic lymphohistiocytosis: experience in adults from a single institution. Ann Hematol 92: 1529-36 (doi: 10.1007/s00277-013-1784-3. Epub 2013 May 23)

43. Buyse S, Teixeira L, Galicier L, Mariotte E, Lemiale V, Seguin A, Bertheau P, Canet E, de Labarthe A, Darmon M, Rybojad M, Schlemmer B, Azoulay E, (2010) Critical care management of patients with hemophagocytic lymphohistiocytosis. Intensive Care Med 36: 1695-1702

44. Aulagnon F, Lapidus N, Canet E, Galicier L, Boutboul D, Peraldi MN, Reuter D, Bernard R, Schlemmer B, Azoulay E, Zafrani L, (2015) Acute kidney injury in adults with hemophagocytic lymphohistiocytosis. Am J Kidney Dis 65: 851-859
45. Créput C, Galicier L, Buyse S, Azoulay E, (2008) Understanding organ dysfunction in hemophagocytic lymphohistiocytosis. Intensive Care Med 34: 1177-1187

46. Fitzgerald NE, McClain KL, (2003) Imaging characteristics of hemophagocytic lymphohistiocytosis. Pediatr Radiol 33: 392-401

47. Santoriello D, Hogan J, D'Agati VD, (2016) Hemophagocytic syndrome with histiocytic glomerulopathy and intraglomerular hemophagocytosis. Am J Kidney Dis 67: 978-983

48. Ramanan AV, Rosenblum ND, Feldman BM, Laxer RM, Schneider R, (2004) Favorable outcome in patients with renal involvement complicating macrophage activation syndrome in systemic onset juvenile rheumatoid arthritis. J Rheumatol 31: 2068-2070

49. Thaunat O, Delahousse M, Fakhouri F, Martinez F, Stephan JL, Noël LH, Karras A, (2006) Nephrotic syndrome associated with hemophagocytic syndrome. Kidney Int 69: 1892-1898

50. Bae MN, Kwak DH, Park SJ, Choi BS, Park CW, Choi YJ, Lee JW, Yang CW, Kim YS, Chung BH, (2016) Acute kidney injury induced by thrombotic microangiopathy in a patient with hemophagocytic lymphohistiocytosis. BMC Nephrol 17: 4

51. Strauss R, Neureiter D, Westenburger B, Wehler M, Kirchner T, Hahn EG, (2004) Multifactorial risk analysis of bone marrow histiocytic hyperplasia with hemophagocytosis in critically ill medical patients - a post mortem clinicopathologic analysis. Crit Care Med 32: 1316-1321

52. Grangé S, Buchonnet G, Besnier E, Artaud-Macari E, Beduneau G, Carpentier D, Dehay J, Girault C, Marchalot A, Guerrot D, Tamion F, (2016) The use of ferritin to identify critically ill patients with secondary hemophagocytic lymphohistiocytosis. Crit Care Med 44: e1045-e1053

53. Machowicz R, Janka G, Wiktor-Jedrzejczak W (2017) Similar but not the same: differential diagnosis of HLH and sepsis. Crit Rev Oncol Hematol 114: 1-12 